\title{
Molecular Magnetic Switches
}

\author{
Daniel Čavlovićs* and Michal Juríček* \\ §SCS-DSM Award for best poster presentation in Organic Chemistry
}

\begin{abstract}
The design and synthesis of molecular switches is of growing importance considering the steep increase in the production of consumer electronics in the recent years. The function of these devices is based on binary electronic circuits and can be achieved by use of bistable magnetic materials. This article reviews four types of molecular systems, which can be switched between two spin states in response to an external stimulus, illustrating working principles that can motivate the design of systems for practical applications. As an outlook, organic diradicaloid molecules are introduced as potential molecular magnetic switches that do not rely on the use of metals.
\end{abstract}

Keywords: Bistability · Magnetic switch · Molecular memory · Spin-crossover $\cdot$ Stimuli-responsive systems

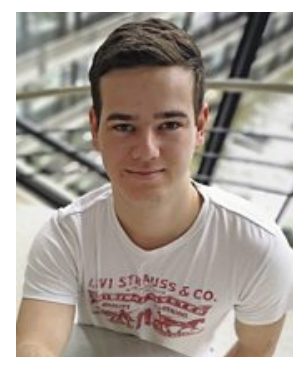

Daniel Čavlović was born in Basel, where he studied chemistry and was awarded his Bachelor's degree in 2016. He continued his Master studies at the University of Basel, but joined the group of Michal Juríček, who just got a position at the University of Zurich, for an external Master thesis. After receiving his Master's degree in 2018, he decided to stay in the Juríček group as a $\mathrm{PhD}$ student and continue his research at the University of Zurich. Daniel designs and synthesizes helical polyaromatic diradicals and diradicaloids and investigates their potential use as magnetic switches.

\section{Introduction}

Innovation of electronic devices, which fulfil the needs of our society, strongly relies on continuous development of novel technologies or materials that make it possible to decrease the size and improve the performance of these devices. The function of a device $^{[1-3]}$ that can store or process data is based on binary electronic circuits, which require bistable materials that can be switched reversibly between two distinct magnetic and conductive states, by an external stimulus. As the demand for downsizing of devices is continuously rising, there is a quest for the smallest possible binary unit, which can perform equally well or better than the current ones. Development of bistable molecules that can be switched between two different magnetic states represents one approach to address this challenge. Various types of molecular materials, the specific properties of which can be turned on and off upon applying a trigger have been developed. ${ }^{[4]}$ Most of these systems, however, function in the bulk, ${ }^{[5]}$ where intermolecular interactions between individual molecules result in stimuli-responsive bistabilities. In this article, a representative selection of known examples that illustrate different working principles for switching magnetic properties within a molecule are presented.

In the literature, molecular magnetic switches have been referred to in several different ways - spin-crossover molecules, spin-switch molecules, or bistable molecular switches, to name

${ }^{*}$ Correspondence: D. Čavlović, Prof. Dr. M. Juríček University of Zurich, Department of Chemistry

Winterthurerstrasse 190, CH-8057 Zurich, Switzerland

E-mail: daniel.cavlovic@chem.uzh.ch,michal.juricek@chem.uzh.ch

Twitter @michal_juricek a few - regardless of the type of their working principle. In this overview, we classify selected examples depending on the type of external stimulus that is employed to trigger the switching process. Four different triggers, namely, (1) mechanical force, (2) electric field, (3) $\mathrm{pH}$, and (4) light are included, out of which the light trigger represents the least invasive triggering technique. Almost all known examples are metal-based and rely on the geometry changes related to metal-ligand coordination. A rather rare exception are diradicaloid polyaromatic hydrocarbons composed of carbon and hydrogen atoms only, which do not contain metals, yet can possess magnetic properties and hold potential to act as metal-free magnetic switches. One example, which illustrates the working principles of making such a molecular magnetic switch, is described in the last section.

\section{Mechanical Trigger}

A representative example of a mechanically triggered magnetic switch was published[6] in 2016 by Mayor, van der Zant, and coworkers. In their system, the mechanical stretching of a terpyridinebased metal complex triggered a transition from a low-spin $(S=$ $0)$ to a high-spin $(S=2)$ state (Fig. 1). The switching between the two states was achieved by geometrical distortion of the coordination sphere induced by a mechanical force, as each geometry favors a different spin ground state. The switch was described as a spin-crossover ${ }^{[7]}$ (SCO) molecule bearing an iron(II) metal center and two phenyl-extended terpyridine ligands, each decorated with a thiol functionality as a gold-anchoring group. Mechanically, controlled break junctions (MCBJ) were prepared from a gold nanowire by a slow controlled stretching, which induced a rupture of the wire. The SCO molecule was incorporated into the junction and conductivity through the molecule was measured under vacuum at cryogenic temperatures $(\sim 6 \mathrm{~K})$ as a function of the distance between the electrodes. When the distance exceeded $0.35 \mathrm{~nm}$, a jump in conductivity to higher current values (1-2 orders of magnitude) was observed, indicating that geometrical distortion caused transition from the low-spin to the high-spin state. A control experiment was performed by using ruthenium(II) instead of iron(II). Because the crystal field of ruthenium, compared to iron, is considerably larger and not as prone to undergo a spin-crossover transition under geometrical distortion, this experiment confirmed their hypothesis, which was further supported by DFT calculations. 

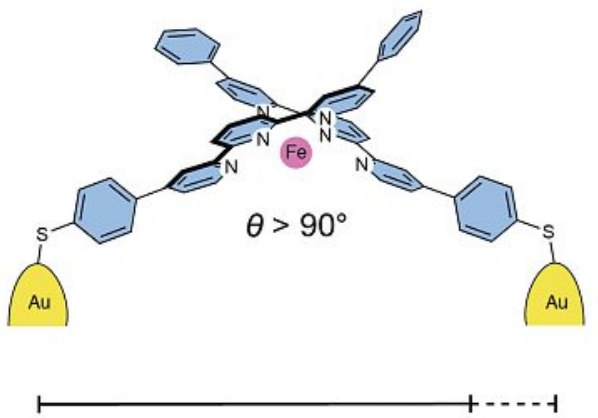

pull $\uparrow$ push

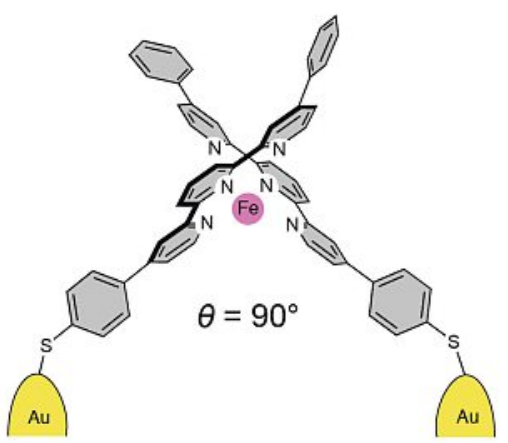

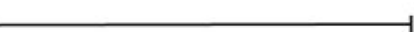

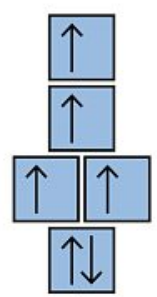

$S=2$

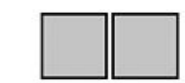

$S=0$
Fig. 1. An iron(II)-based spin-switch anchored in a mechanically controlled break junction that can be switched between a low-spin $(S=0)$ and a high-spin $(S=2)$ state by using mechanical force. The coordination bonds Fe-N are omitted for clarity.

\section{Electric-Field Trigger}

The same collaborating groups of Mayor and van der Zant reported $^{[8]}$ in 2015 a conceptually different SCO switch based on a terpyridine iron(II) complex (Fig. 2). In this system, the spinswitching principle, namely, geometrical distortion of the coordination sphere, is the same as in the previous example, but the switching is triggered by applying an electric field. This switch features a heteroleptic iron(II) complex. One ligand acts as a bridge and fixes the molecule in the molecular junction. The second, perpendicular ligand possesses a distinct dipole moment and can sense an applied electric field, which forces the ligand to partially align in the direction of the electric field. The alignment causes coordinational distortion and is dependent on the field strength. The electric-field sensing was achieved by installing one donor and one acceptor substituent at each end of the non-bridging ligand. A series of donor-acceptor complexes were prepared with dipole moments of varying magnitude. The conductivity through these molecules was measured as a function of applied voltage. Although these measurements showed voltage-dependent bistability of the systems, none of them supported an ideal scenario, which would be a significant increase in conductivity at a certain voltage threshold, both at a positive and a negative bias.

\section{4. $\mathrm{pH}$ Trigger}

An example of a magnetic switch triggered by changes in $\mathrm{pH}$ was reported ${ }^{[9]}$ by Sawaki and co-workers in 1997, who introduced a cross-conjugated phenyloxy-nitronyl nitroxide diradical (Fig. 3 ). This switch represents a rather uncommon example of a nonmetal-containing organic molecule that can be switched between
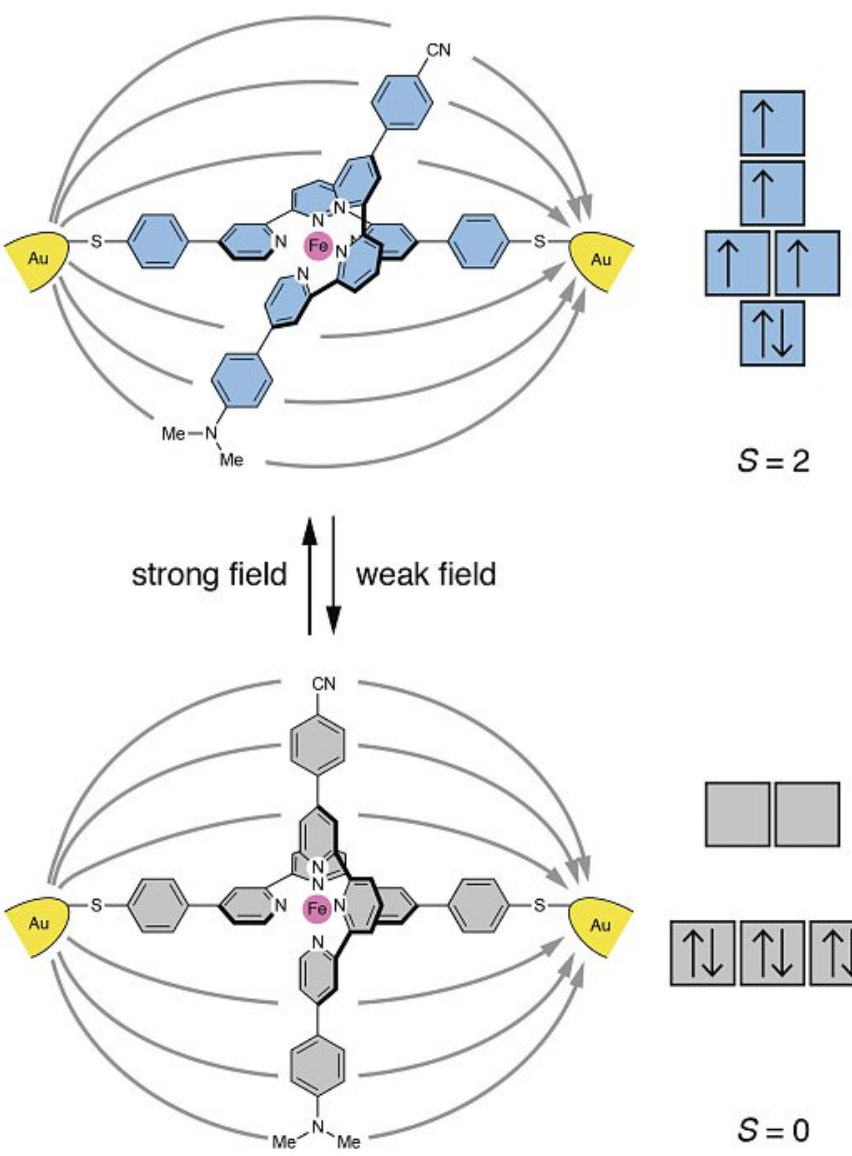

Fig. 2. An iron(॥)-based spin-switch anchored in a mechanically controlled break junction that can be switched between a low-spin $(S=0)$ and a high-spin $(S=2)$ state by using electric field. The coordination bonds Fe-N are omitted for clarity.

two spin states. Both forms of this switch can be accessed from the same phenol precursor, depending on the extent of oxidation. One-electron oxidation gives the open-shell diradical form, while two-electron oxidation gives the closed-shell form. Both forms can interconvert to one another upon the addition of acid or base. The deprotonated open-shell form has a triplet ground state $(S=1)$ and it showed high stability in solution, which allowed its isolation

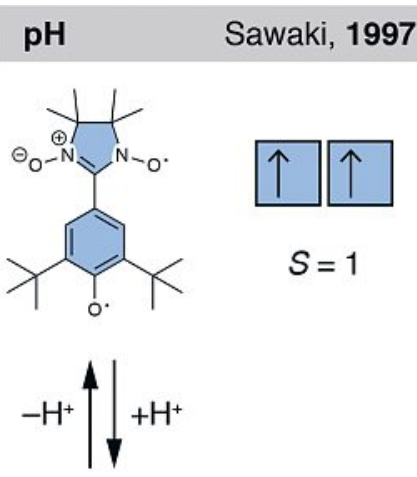

Fig. 3. An all-organic spin-switch that can be switched between a low-spin $(S=0)$ and a high-spin $(S=1)$ state by changing $\mathrm{pH}$. Note: In the original work, it was not specified whether the singly occupied molecular orbitals of the deprotonated form are degenerate or not. They are shown as degenerate for simplicity.<smiles>CC(C)(C)c1cc(C2=[N+]([O-])C(C)(C)C(C)(C)[N+]2=O)cc(C(C)(C)C)c1O</smiles>

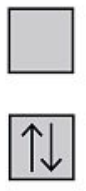

$S=0$ 
as a solid powder. The protonated closed-shell form has a singlet ground state $(S=0)$ and a $\mathrm{p} K_{\mathrm{a}}$ in the range 10-11 (experimental values). The switching process can be monitored by means of electron paramagnetic resonance (EPR) spectroscopy, whereby the protonated form is EPR-silent and the deprotonated form is EPRactive, a feature that makes this switch useful for applications as a $\mathrm{pH}$-sensor responding by changes in magnetic properties.

\section{Light Trigger}

The switches presented above have certain limitations. They either require vacuum, cryogenic temperatures, or chemical manipulation ( $\mathrm{pH}$ alteration). The last type of systems presented in this article, namely, light-triggered molecular magnetic switches, are less invasive and have additional advantages, such as high speed of switching and wavelength specificity. Three examples that have different chemical working principles are presented.

In 2011, Herges and co-workers reported[10] a light-triggered molecular magnetic switch, the working principle of which is based on changing the coordination number of a nickel(II) porphyrin complex, and thereby the spin ground state, through cis-trans photoisomerization of a phenylazopyridine moiety (Fig. 4). This switch is reminiscent of a record player, where the square planar nickel(II) porphyrin complex acts as the turntable housing and the phenylazopyridine moiety acts as a pickup arm bearing the lone electron pair of the pyridine unit as a needle. In the cis form, the pyridine unit is coordinated to the nickel center, which has a coordination number 5 and an open-shell electronic structure $(S=1)$. In the trans form, the pyridine unit is too far to be coordinated and the nickel center has a coordination number 4 and a closed-shell electronic structure $(S=0)$. Typically, the $c i s$ isomers of azo compounds are rather unstable and can thermally interconvert to the trans isomers. In this case, however, the cis form is stabilized due to coordination of the pyridine unit to the metal center and displays a remarkable stability. Concentrationdependent Evans measurements confirmed that the cis form is paramagnetic and that the switching results from the intramolecular isomerization and not from an intermolecular coordination. In a control experiment, an analogous zinc(II) complex remained diamagnetic upon switching from the trans to the cis form, as zinc(II) has a $d^{10}$ configuration and not $d^{8}$ like nickel(II). Prior to Herges' switch, magnetic bistability was restricted to bulk materials or very low temperatures. This switch, however, is stable in a homogeneous solution at room temperature, is fully reversible, and shows no fatigue or degradation even after several thousand cycles. In addition, preliminary experiments show the potential of this switch to be used as a photoswitchable magnetic resonance imaging (MRI) contrast agent.

Another example of a light-triggered magnetic switch was reported ${ }^{[11]}$ by Khusniyarov and co-workers in 2013 (Fig. 5). In contrast to Herges' system, the metal center of Khusniyarov's switch retains its coordination number upon switching and its spin state is altered by the ligand, the electronic structure of which is changed upon light irradiation. In this system, a photoswitchable dithienylethene-based ligand was incorporated into an SCO iron(II) complex containing boron-bridged pyrazolyl ligands. Upon irradiation, the open form of the photoactive ligand undergoes a photocyclization reaction to the closed form, a process that can be reversed upon irradiation at another wavelength. The ligandfield strength is different in the two forms, which leads to distinct spin preferences, namely, $S=2$ in the open form and $S=0$ in the closed form, and thus to an SCO upon switching. Both forms were thermally stable at room temperature with a half-time of 18 days in solution and the switching could be monitored by measuring the effective magnetic moment using Evans method.

Last year, our group reported ${ }^{[12]}$ a helicene-based chiroptical photoswitch, the working principle of which can be employed in the design of magnetic switches (Fig. 6). Unlike the above ex-
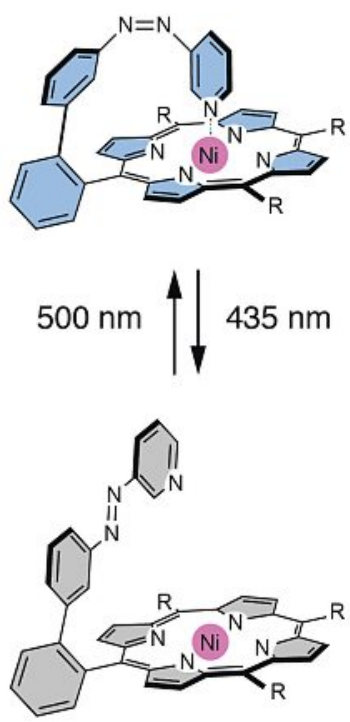

$\mathrm{R}=\mathrm{C}_{6} \mathrm{~F}_{5}$

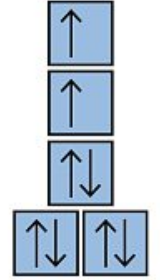

$S=1$

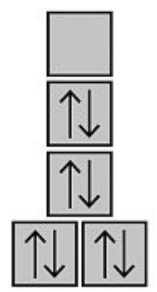

$S=0$
Fig. 4. A nickel(II)-based spin-switch that can be switched between a low-spin $(S=0)$ and a high-spin $(S=1)$ state by light irradiation, which triggers photoisomerization of the azo double bond and changes the coordination number of the metal center from 4 in the trans isomer to 5 in the cis isomer. The coordination bonds $\mathrm{Ni}-\mathrm{N}$ are omitted for clarity.
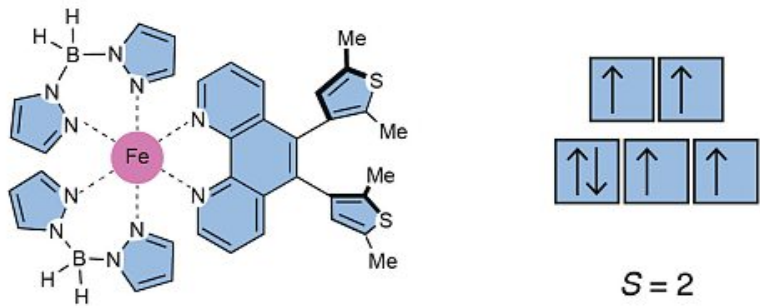

\section{$S=2$}
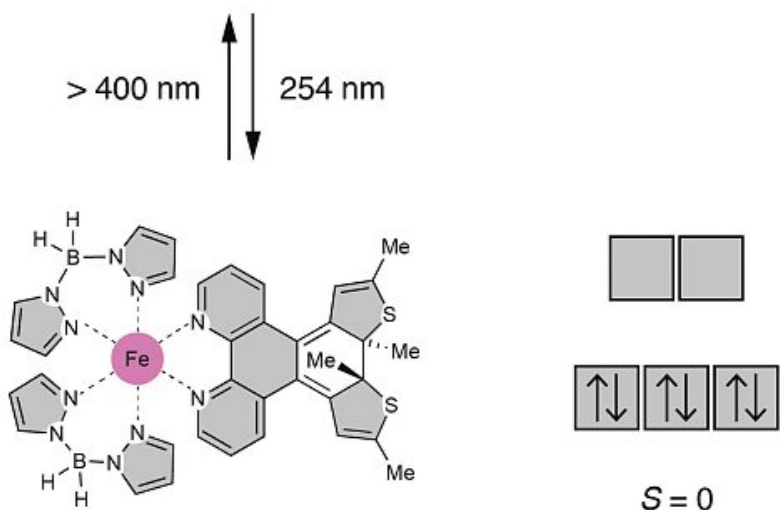

Fig. 5. An iron(II)-based spin-switch that can be switched between a lowspin $(S=0)$ and a high-spin $(S=2)$ state by light irradiation, which triggers electrocyclic ring closure/opening of one of the ligands. The open and the closed form of the ligand each favors a different spin state.

amples, this system is composed of carbon and hydrogen atoms only. The open form of the switch is a helically chiral polyaromatic hydrocarbon, composed of seven fused benzenoid rings, that we named ${ }^{[13]}$ cethrene after its $\mathrm{C}$-shape and chirality. Similarly to Khusniyarov's switch, cethrene also contains a hexatriene subunit and can undergo a reversible electrocyclic ring closure ${ }^{[14,15]}$ 
to the closed form. This process can be triggered by light in both directions and substantially alters the HOMO-LUMO and singlet-triplet energy gaps. While the energy gaps in the closed form are relatively large, the open form possesses very small HOMOLUMO and singlet-triplet energy gaps ${ }^{[16]}$ and has a diradicaloid ${ }^{[17]}$ character. In general, if the singlet-triplet gap is sufficiently low, the triplet state can be populated thermally at room temperature, which can give rise to paramagnetic properties in a formally singlet closed-shell molecule. Although in the case of the dimethyl derivative shown in Fig. 6, the singlet-triplet gap is too high to observe an EPR signal at room temperature, this system demonstrates the concept that helical diradicaloids could be employed in the design of magnetic switches.

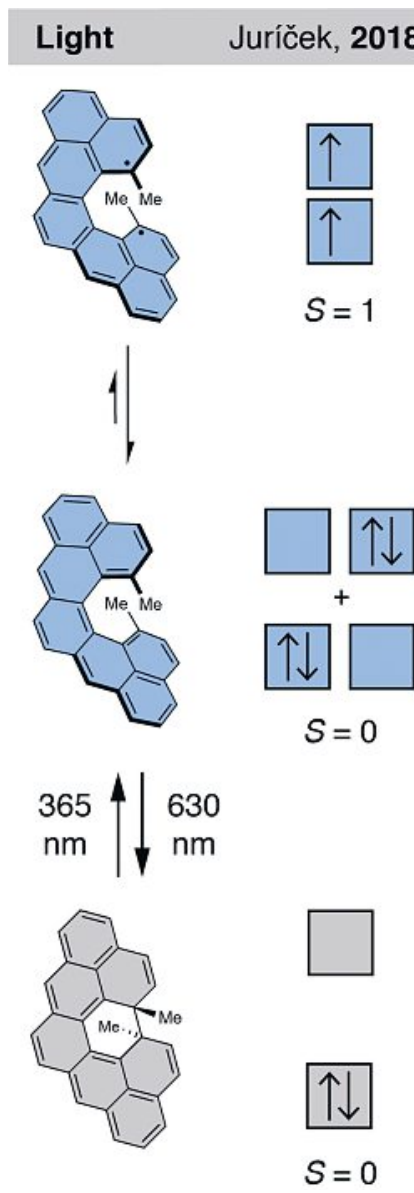

\section{Conclusion \& Outlook}

Five representative examples of molecular magnetic switches -were presented in the context of their working principles and stimuli to trigger the spin-switching process. Although some systems require operating conditions, which limit their potential for practical applications, such as vacuum, low temperatures, or chemical treatment, all examples beautifully demonstrate that there is no limit to chemical design and imagination. While most of the known magnetic switches are based on metal complexes, some examples illustrate that spin-switching can be achieved also in non-metal-based system. Our group has demonstrated that helical diradicaloid systems hold the potential to be employed in the design of magnetic photoswitches and is currently optimizing the molecular design to validate this concept.

\section{Acknowledgements}

This project received funding from the European Research Council (ERC) under the European Union's Horizon 2020 research and innovation programme (Grant Agreement No. 716139) and the Swiss National Science Foundation (SNSF, M.J./ PP00P2_170534). D.Č. would like to thank DSM and the Swiss Chemical Society (SCS) for the poster presentation award.

Received: February 8, 2019

[1] K. Szacilowski, 'Infochemistry: Information Processing at the Nanoscale', John Wiley \& Sons, 2012.

[2] J. Bartolomé, F. Luis, J. F. Fernández, 'Molecular Magnets: Physics and Applications', Springer Science \& Business Media, 2013

[3] N. Lorente, C. Joachim, 'Architecture and Design of Molecule Logic Gates and Atom Circuits: Proceedings of the 2nd AtMol European Workshop', Springer Science \& Business Media, 2013.

[4] R. G. Hicks, 'Stable Radicals: Fundamentals and Applied Aspects of OddElectron Compounds', John Wiley \& Sons, 2010.

[5] M. E. Itkis, X. Chi, A. W. Cordes, R. C. Haddon, Science 2002, $296,1443$.

[6] R. Frisenda, G. D. Harzmann, J. A. Celis Gil, J. M. Thijssen, M. Mayor, H. S. J. van der Zant, Nano Lett. 2016, 16, 4733.

[7] P. Gütlich, H. A. Goodwin, 'Spin Crossover in Transition Metal Compounds III', Springer Science \& Business Media, 2004.

[8] G. D. Harzmann, R. Frisenda, H. S. J. van der Zant, M. Mayor, Angew. Chem. Int. Ed. 2015, 54, 13425.

[9] K. Ishiguro, M. Ozaki, N. Sekine, Y. Sawaki, J. Am. Chem. Soc. 1997, 119, 3625.

[10] S. Venkataramani, U. Jana, M. Dommaschk, F. D. Sönnichsen, F. Tuczek, R. Herges, Science 2011, 331, 445.

[11] M. Milek, F. W. Heinemann, M. M. Khusniyarov, Inorg. Chem. 2013, 52, 11585.

[12] P. Ravat, T. Šolomek, D. Häussinger, O. Blacque, M. Juríček, J. Am. Chem. Soc. 2018, 140, 10839.

[13] M. Juríček, Chimia 2018, 72, 322.

[14] P. Ravat, T. Šolomek, M. Juríček, ChemPhotoChem 2019, doi:10.1002/ cptc. 201800229.

[15] T. Šolomek, P. Ravat, Z. Mou, M. Kertesz, M. Juríček, J. Org. Chem. 2018, 83, 4769.

[16] P. Ravat, T. Šolomek, P. Ribar, M. Juríček, Synlett 2016, 27, 1613.

[17] P. Ravat, T. Šolomek, M. Rickhaus, D. Häussinger, M. Neuburger, M. Baumgarten, M. Juríček, Angew. Chem. Int. Ed. 2016, 55, 1183. 